\title{
Analytical Determination of the PZTs Distribution in Active Beam Vibration Protection Problem
}

\author{
A. BRAŃSKI ${ }^{a, *}$ AND G. LIPIŃSKI ${ }^{b}$ \\ ${ }^{a}$ Laboratory of Acoustics, Department of Electrical and Computer Engineering, \\ Rzeszów University of Technology, W. Pola 2, 35-959 Rzeszów, Poland \\ ${ }^{b}$ Department of Mechanical Engineering and Aeronautics, Rzeszów University of Technology \\ W. Pola 2, 35-959 Rzeszów, Poland
}

\begin{abstract}
The paper concerns an active vibration protection (p-reduction) of the structure via piezoelectric transducers; p-reduction corresponds to an active vibration reduction (a-reduction). The quantity and effectiveness of the (a- or p-) reduction, among other parameters, depend on the piezoelectric transducers distribution on the structure. The best results are obtained bonding piezoelectric transducers to the structure in the sub-domains with the largest curvatures; it is so-called quasi-optimal distribution of the piezoelectric transducers. Up to now, the quasi-optimal distribution was determined based on heuristic reasons only. The aim of the paper is to confirm quasi-optimal distribution in analytical way. The beam clamped at one end, vibrating with first three modes separately, is chosen as the research object. It is assumed that the piezoelectric transducers are exactly the same. Demanding the vibration amplitude to be equal to zero (i.e. p-reduction condition), the general formula for interacting forces piezoelectric transducers-beam is derived. Next, such an appropriate distribution of piezoelectric transducers is searched analytically, that the minimal forces are achieved; it leads to the best reduction effectiveness. It turned out that the analytical method pointed out quasi-optimal distribution of the piezoelectric transducers. The validation of theoretical considerations is confirmed numerically.
\end{abstract}

PACS: 43.40.Tm, 43.40.Vn, 77.65.-j, 46.40.-f, 62.25.Jk

\section{Introduction}

An active vibration reduction of the structure (a-reduction) vibrating with low frequencies is generally known $[1,2]$. The active vibration protection (p-reduction) is the particular case of a-reduction [3]. The reduction (a- or p-) is realized with piezoelectric elements, actuators (PZTs).

The quantity of the reduction depends on many factors. The first of them makes up the PZTs' geometric-technical features [4-8]. The second essential factor is appropriate PZTs distribution on the structure [9-11]. It has an influence on different parameters of the structure or on the radiated acoustic field $[12,13]$.

Now, a question arises about an optimal distribution of PZTs. In the recent year, a great number of papers has been published on this subject. It is obvious that there are a lot of optimization techniques; an excellent survey is given in [14] and references given therein. Two main approaches are distinguished there. The former is the coupling of the optimization of actuators/sensors locations and control parameters. As the criterion for the optimization, the following criterions are taken into account:

* corresponding author; e-mail: abranski@prz.rzeszow.pl
- the quadratic cost function of the measure error and the control energy,

- the maximization of dissipation energy during the control,

- the spatial $\mathrm{H}_{2}$ norm of the closed-loop transfer matrix from the disturbance to the distributed controlled output,

- the simultaneous simple $H_{\infty}$ controller,

As can be seen, the optimization criterions are dependent on the choice of controllers. Therefore, the optimal locations obtained using one controller may not be a suitable choice for another one. So at the latter approach, the optimal locations are obtained independently of the controller definition. In this case, the following criterions are used:

- the maximization controllability/observability criterion using the Gramian matrices,

- the modal controllability index based on singular value analysis of the control vector,

- the maximization of the control forces transmitted by the actuators to the structure,

- using the $H_{2}$ norm. 
But in the mentioned references, the PZTs distribution was not provided explicitly, to confirm maximum effectiveness of the vibration reduction. This problem was solved [3, 15-17]. It was proved there that the most effective PZTs distribution is on the structure sub-domains with the largest curvatures; such distribution is called quasi-optimal one (QO). In the quoted papers, the QO-distribution is deduced based on the heuristic reasons.

The aim of this paper is the theoretical derivation of such PZTs distribution, to provide the maximum effectiveness of the p-reduction of the beam separately modes.

The beam clamped at first end and free second one is chosen as the research object. It is assumed that the beam is excited with evenly spread and harmonic force. The force acts with first three natural frequencies separately. The internal damping coefficient of the material is introduced.

It is assumed that all PZTs are identical from the geometrical and technical aspect. As a result, the PZTs interact with the beam with the same forces. The forces values depend on the number and the distribution of PZTs on the beam. To determine them, the following algorithm is used. First, applying the p-reduction condition, the formula on the forces (amplitude, strictly speaking) is derived; the necessary condition of the p-reduction is the vibration amplitude to be equal to zero. Next, establishing the number of PZTs (not by coincidence) and considering the different distribution of the PZTs, the minimal values of forces are searched theoretically. This means that the minimum energy is added to the system and consequently, the maximum effectiveness of the p-reduction is assured. An effectiveness measure of the reduction is an effectiveness coefficient; it is defined in $[3,15,16]$.

To the authors' knowledge recently, the theoretical description of the PZTs distribution on even simple structure like the beam, have not been considered. The validity of theoretical considerations is confirmed numerically. It turned out that the PZTs distribution theoretically found in the paper is identical to that one found on the heuristic way.

\section{Forced vibration of the beam with damping}

Let the beam be clamped at one side, Fig. 1; geometrical data of the beam: $\ell$ - length, $S=a h$ - surface of the rectangular cross-section, $a$ - width, $h$ - thickness. Moreover, $q_{\mathrm{E}}=q_{\mathrm{E}}(x, t)$ - excited force. The beam vibration equation is given by [18] p. 172, [19] p. 104, [5] p. 27, [20] p. 238,

$$
E J\left[D_{x}^{4} u+\mu D_{x}^{4}\left(D_{t} u\right)\right]+S D_{t}^{2} u=-q_{\mathrm{E}},
$$

where $u=u(x, t)$ - beam deflection at the point $x$ and the moment $t, E-$ Young's modulus, $J$ - surface moment of inertia of the beam cross-section, $\rho$ mass density, $\mu$ - internal damping factor, $D_{x}^{4}(\ldots)=$ $\partial^{4}(\ldots) / \partial x^{4}, D_{t}(\ldots)=\partial(\ldots) / \partial t$.

The boundary conditions are described by the following equations:

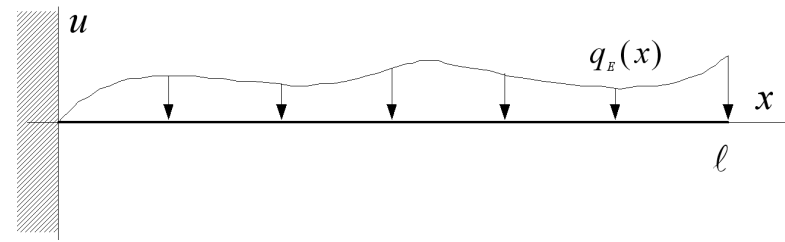

Fig. 1. The geometry of the problem.

$$
\begin{aligned}
& u(x=0, t)=0, \quad D_{x} u(x=0, t)=0, \\
& D_{x}^{2} u(x=\ell, t)=0, \quad D_{x}^{3} u(x=\ell, t)=0 .
\end{aligned}
$$

Besides, initial conditions are assumed to be equal to zero. The solution of the formulated problem is forced harmonic vibrations with damping. Let be the lateral load force

$$
q_{\mathrm{E}}(x, t)=q_{\mathrm{E}}(x) \exp \left(\mathrm{i} \omega_{f} t\right),
$$

where $\mathrm{i}=(-1)^{1 / 2}, \omega_{f}$ - frequency.

Applying eigenfunction expansion method, the solution of Eq. (1) is assumed as

$$
u(x, t)=X(x) \exp \left(\mathrm{i} \omega_{f} t\right) .
$$

After some calculation, the solution of the above problem is

$$
\begin{gathered}
X(x)=X_{f}(x)=\sum_{\nu} C_{\nu} X_{\nu}(x)=\sum_{\nu} X_{f ; \nu}(x), \\
\nu=1,2, \ldots, n,
\end{gathered}
$$

where $C_{\nu}$ - any constants, $X_{f}(x)$ - forced vibrations, $X_{\nu}(x)-\nu$-modes (eigenfunctions),

$$
\begin{gathered}
X_{\nu}(x)=c\left(\lambda_{\nu} \ell\right)\left[\sinh \left(\lambda_{\nu} x\right)-\sin \left(\lambda_{\nu} x\right)\right] \\
-s\left(\lambda_{\nu} \ell\right)\left[\cosh \left(\lambda_{\nu} x\right)-\cos \left(\lambda_{\nu} x\right)\right], \\
c\left(\lambda_{\nu} \ell\right)=\cos \left(\lambda_{\nu} \ell\right)+\cosh \left(\lambda_{\nu} \ell\right), \\
s\left(\lambda_{\nu} \ell\right)=\sin \left(\lambda_{\nu} \ell\right)+\sinh \left(\lambda_{\nu} \ell\right),
\end{gathered}
$$

$\left\{\lambda_{\nu}\right\}$ - set of eigenvalues: $\left\{\lambda_{\nu} \ell\right\}=\{1.8751,4.6941$, $\ldots,(2 \nu-1) \pi / 2\}$.

The constants $C_{\nu}$ are expressed by

$$
\begin{aligned}
C_{\nu} & =\frac{1}{\left(1+\mathrm{i} \mu \omega_{f}\right) \omega_{\nu}^{2}-\omega_{f}^{2}} D_{\nu}=\frac{1}{\alpha_{\nu}^{2}} D_{\nu} \\
& =\frac{1}{\rho S} \frac{1}{\alpha_{\nu}^{2}} \frac{1}{\beta_{\nu}^{2}} I_{\nu ; \mathrm{E}}=C_{\nu}^{*} I_{\nu ; \mathrm{E}}, \\
C_{\nu}^{*} & =\frac{1}{\rho S} \frac{1}{\alpha_{\nu}^{2}} \frac{1}{\beta_{\nu}^{2}}, \quad I_{\nu ; \mathrm{E}}=-\int_{0}^{\ell} q_{\mathrm{E}} X_{\nu}(x) \mathrm{d} x, \\
\omega_{\nu}^{2} & =\frac{E J}{\rho S} \lambda_{\nu}^{4}, \quad \beta_{\nu}^{2}=\int_{0}^{\ell} X_{\nu}^{2}(x) \mathrm{d} x .
\end{aligned}
$$

Thus, the problem of the beam vibration with damping, excited with the force $q_{\mathrm{E}}(x, t)$ is solved. The first three modes, Eq. (7), are depicted in Fig. 2.

Henceforth, the spread load force with constant amplitude $q_{\mathrm{E}}$ is considered, i.e.

$$
q_{\mathrm{E}}(x)=q_{\mathrm{E}}=\text { const. }
$$




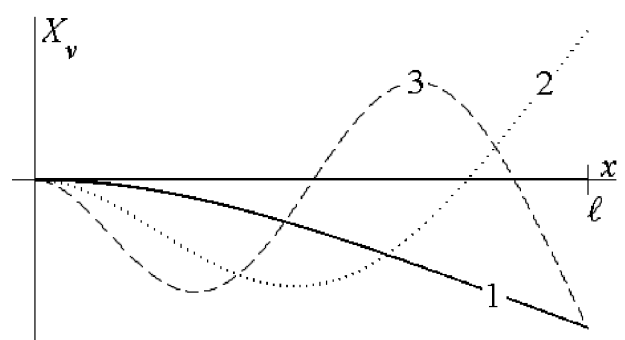

Fig. 2. Eigenfunctions (modes): $1-X_{1}, 2-X_{2}$, $3-X_{3}$.

\section{Beam vibration reduction by PZT}

As it is already known [1, 2], the PZTs-beams interact with moments of the couples of forces, Fig. 3a. Since the beam vibration equation is the forces equation, then to consider the action of PZTs on the beam, two moments are replaced with two couples of forces, Fig. 3b. Next, the separate forces are taken into account in Eq. (1). Hence, the total load is the sum of the load force expressed by Eq. (12) and the forces interacting between PZTs and the beam, and it is given by

$$
\begin{aligned}
& f(x)=-q_{\mathrm{E}}+\left[f_{\mathrm{P}} \delta\left(x_{\mathrm{P}}-l_{\mathrm{P}}\right)-2 f_{\mathrm{P}} \delta\left(x_{\mathrm{P}}\right)\right. \\
& \left.\quad+f_{\mathrm{P}} \delta\left(x_{\mathrm{P}}+l_{\mathrm{P}}\right)\right] .
\end{aligned}
$$

An expression in the bracket is the sum of interacting forces PZTs-beam, $\delta($.$) - the Dirac function.$

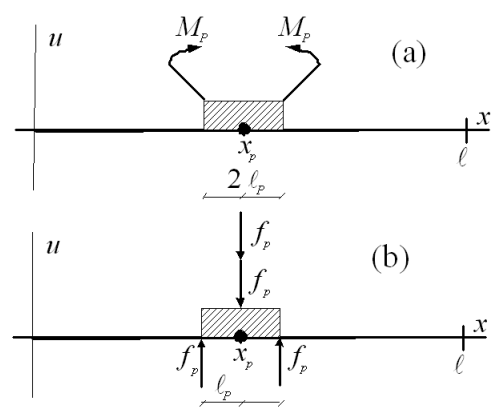

Fig. 3. An idea of interaction of the PZT-beam: (a) moments, (b) couples of forces.

The integral $I_{\nu}$, Eq. (11), for $f(x)$ given by Eq. (13), takes the form

$$
\begin{aligned}
I_{\nu} & =-\int_{0}^{\ell} f(x) X_{\nu}(x) \mathrm{d} x=-q_{\mathrm{E}} \int_{0}^{\ell} X_{\nu}(x) \mathrm{d} x \\
& +f_{\mathrm{P}} \int_{0}^{\ell}\left(\delta\left(x_{\mathrm{P}}-l_{\mathrm{P}}\right)-2 \delta\left(x_{\mathrm{P}}\right)+\delta\left(x_{\mathrm{P}}+l_{\mathrm{P}}\right)\right) \\
& \times X_{\nu}(x) \mathrm{d} x=-q_{\mathrm{E}} \int_{0}^{\ell} X_{\nu}(x) \mathrm{d} x+f_{\mathrm{P}}\left[X_{\nu}\left(x_{\mathrm{P}}-l_{\mathrm{P}}\right)\right. \\
& \left.-2 X_{\nu}\left(x_{\mathrm{P}}\right)+X_{\nu}\left(x_{\mathrm{P}}+l_{\mathrm{P}}\right)\right] .
\end{aligned}
$$

The expression in square bracket constitutes the second-order central finite difference. Since the distance be- tween nodes $l_{\mathrm{P}}$ is constant, then the difference can be transformed into

$$
\begin{aligned}
& \frac{1}{l_{\mathrm{P}}^{2}}\left[X_{\nu}\left(x_{\mathrm{P}}-l_{\mathrm{P}}\right)-2 X_{\nu}\left(x_{\mathrm{P}}\right)+X_{\nu}\left(x_{\mathrm{P}}+l_{\mathrm{P}}\right)\right] \\
& \quad=D_{x}^{2} X_{\nu}\left(x_{\mathrm{P}}\right)=\kappa_{\nu}\left(x_{\mathrm{P}}\right)
\end{aligned}
$$

where $\kappa_{\nu}\left(x_{\mathrm{P}}\right)$ is the curvature of the mode $X_{\nu}(x)$ at the point $x=x_{\mathrm{P}}[3,15,16]$.

Generally

$$
\kappa_{\nu}(x)= \pm D_{x}^{2} X_{\nu}(x) \text {. }
$$

Substituting Eq. (15) into Eq. (14), one obtains

$$
I_{\nu}=-q_{\mathrm{E}} \int_{0}^{\ell} X_{\nu}(x) \mathrm{d} x+f_{\mathrm{P}} l_{\mathrm{P}}^{2} \kappa_{\nu}\left(x_{\mathrm{P}}\right)=I_{\nu ; \mathrm{E}}+I_{\nu ; P} .
$$

For several PZTs, instead of Eq. (17), is

$$
\begin{aligned}
I_{\nu} & =I_{\nu ; \mathrm{E}}+f_{1} \ell_{1}^{2} \kappa_{\nu}\left(x_{1}\right)+f_{2} \ell_{2}^{2} \kappa_{\nu}\left(x_{2}\right)+\ldots \\
& =I_{\nu ; \mathrm{E}}+\sum_{\mathrm{P}} f_{\mathrm{P}} \ell_{\mathrm{P}}^{2} \kappa_{\nu}\left(x_{\mathrm{P}}\right)=I_{\nu ; \mathrm{E}}+I_{\nu ; \Sigma}, \\
& P=1,2, \ldots, n_{\mathrm{P}},
\end{aligned}
$$

where $n_{\mathrm{P}}$ - number of PZTs, $\left\{x_{\mathrm{P}}\right\}$ - set of the points where PZTs are bonded; both $n_{\mathrm{P}}$ and $\left\{x_{\mathrm{P}}\right\}$ should be determined; it is the aim of the paper.

Substituting Eq. (10) into Eq. (6) via Eq. (17), the reduction vibration is obtained

$$
\begin{aligned}
& X_{f}(x)=\sum_{\nu} C_{\nu}^{*} I_{\nu} X_{\nu}(x)=\sum_{\nu} C_{\nu}^{*}\left(I_{\nu ; \mathrm{E}}+I_{\nu ; \Sigma}\right) X_{\nu}(x) \\
& \quad=\sum_{\nu} A_{f ; \nu} X_{\nu}(x) .
\end{aligned}
$$

Together with the vibration reduction amplitude $A_{f ; \nu}$, the curvature is subjected to reduction and instead of Eq. (16) is

$$
\begin{aligned}
& \kappa_{f}(x)= \pm D_{x}^{2} X_{f}(x)=\sum_{\nu} \kappa_{f ; \nu}(x) \\
& = \pm \sum_{\nu} C_{\nu}^{*}\left(I_{\nu ; \mathrm{e}}+I_{\nu ; \Sigma}\right) \kappa_{\nu}(x)= \pm \sum_{\nu} A_{f ; \nu} \kappa_{\nu}(x) .
\end{aligned}
$$

The reduction of the $A_{f ; \nu}$ leads to the reduction of the shear force $Q(x)$ and bending moment $M(x)[3,18]$ p. 172, [13],

$$
\begin{aligned}
& Q_{f}(x)= \pm E J D_{x} \kappa_{f}(x)= \pm E J \sum_{\nu} D_{x} \kappa_{f ; \nu}(x) \\
& = \pm E J \sum_{\nu} A_{f ; \nu} D_{x} \kappa_{\nu}(x) \\
& M_{f}(x)=\mp E J \kappa_{f}(x)= \pm E J \sum_{\nu} \kappa_{f ; \nu}(x) \\
& \quad= \pm E J \sum_{\nu} A_{f ; \nu} \kappa_{\nu}(x) .
\end{aligned}
$$

From the last equations it appears that the active vibration reduction undergoes on the following amplitudes: beam vibration $X_{f}(x)$ - Eq. (19), shear force $Q_{f}(x)$ Eq. (21) and bending moment $M_{f}(x)-$ Eq. (22). This is the same amplitude $A_{f ; \nu}$, so it may be named as the reduction amplitude. Hereafter, the notion "shear force 
reduction" is used instead of "the reduction of the amplitude of the shear force", and so on.

Equations (19), (21) and (22) may be written commonly

$$
\Psi_{f}(x)=\sum_{\nu} \Psi_{f ; \nu}(x)=C \sum_{\nu} A_{f ; \nu} \Phi_{\nu}(x),
$$

where (argument $x$ is omitted)

$$
\begin{aligned}
& \Psi_{f}=\left\{X_{f}, Q_{f}, M_{f}\right\}, \quad \Phi_{\nu}=\left\{X_{\nu}, D_{x} \kappa_{\nu}, \kappa_{\nu}\right\}, \\
& C=\{1, \pm E J, \pm E J\} .
\end{aligned}
$$

Let us note that the amplitude $A_{f ; \nu}$ is the direct quantity which is liable to the reduction, in explicit form is

$$
\begin{aligned}
& A_{f ; \nu}=C_{\nu}^{*}\left(I_{\nu ; \mathrm{E}}+I_{\nu ; \Sigma}\right) \\
& \quad=C_{\nu}^{*}\left[I_{\nu ; \mathrm{E}}+\sum_{\mathrm{P}} f_{\mathrm{P}} \ell_{\mathrm{P}}^{2} \kappa_{\nu}\left(x_{\mathrm{P}}\right)\right] .
\end{aligned}
$$

\section{4. p-reduction problem, solution with heuristic method}

As can be seen from Eq. (23), the reduction of $A_{f ; \nu}$ brings about the reduction of $\Psi_{f}(x)$. The general classification of the reduction is as follows [16]:

- a-reduction; [a]ctive,

- p-reduction; [p]rotection.

a-reduction of $\Psi_{f}(x)$ means decreasing of the quantity $\Psi_{f}(x)$. Hence, it is demand to fulfill the following condition: $\Psi_{f}=\Psi_{f ; \min }$. So, it leads to the condition: $A_{f ; \nu}=A_{f ; \nu ; \min }$.

p-reduction is a special case of the a-reduction and it means the protection of $\Psi_{f}(x)$. Therefore it leads to the condition $\Psi_{f}=0$ or

$$
A_{f ; \nu}=0 \text {. }
$$

The amplitude $A_{f ; \nu}$ comprises the factor $C_{\nu}^{*} \neq 0$ and it is constant. In practice, instead of the condition (26), the following condition of the p-reduction must be fulfilled:

$$
I_{\nu ; \mathrm{E}}+I_{\nu ; \Sigma}=I_{\nu ; \mathrm{E}}+\sum_{\mathrm{P}} f_{\mathrm{P}} \ell_{\mathrm{P}}^{2} \kappa_{\nu}\left(x_{\mathrm{P}}\right)=0 .
$$

The sign of $I_{\nu ; \mathrm{E}}$ is negative, see Eq. (17). To fulfill the condition (27), the sign of $I_{\nu ; \Sigma}$ must be positive. The signs of the curvature $\kappa_{\nu}\left(x_{\mathrm{P}}\right)$ and the forces $f_{\mathrm{P}}$ decide on the sign $I_{\nu ; \Sigma}$. From the technical and reduction effectiveness points of view, the condition (27) should be fulfilled as little as possible number $n_{\mathrm{P}}$ (number of PZTs). It is possible if the signs of $\kappa_{\nu}\left(x_{\mathrm{P}}\right)$ and $f_{\mathrm{P}}$ are the same, namely positive or negative. An idea of description of the signs of $\kappa_{\nu}\left(x_{\mathrm{P}}\right)$ and $f_{\mathrm{P}}$ arise from the physical interpretation of the active vibration reduction; it is so-called the signs convention and is described in detail in $[3,15,16]$.

Assume that all PZTs are exactly the same, hence $\left\{f_{\mathrm{P}}\right\}=\left\{f_{0}\right\}$ and $\left\{\ell_{\mathrm{P}}\right\}=\left\{\ell_{0}\right\}$. So, instead of Eq. (27) one has

$$
I_{\nu ; \mathrm{E}}+I_{\nu ; \Sigma}=I_{\nu ; \mathrm{E}}+f_{0} \ell_{0}^{2} K_{\nu}=0,
$$

$$
\begin{aligned}
& K_{\nu}=\sum_{\mathrm{P}} \kappa_{\nu}\left(x_{\mathrm{P}}\right)=\kappa_{\nu}\left(x_{1}\right)+\kappa_{\nu}\left(x_{2}\right)+\ldots \\
& \quad+\kappa_{\nu}\left(x_{\mathrm{P}}\right)+\ldots+\kappa_{\nu}\left(x_{n_{\mathrm{P}}}\right) .
\end{aligned}
$$

$K_{\nu}$ means the sum of curvatures of the modes $X_{\nu}(x)$ at the points in which the PZTs are bonded (at the $x_{\mathrm{P}}$ points). From Eq. (29) it is derived

$$
f_{0}=-\frac{I_{\nu ; E}}{\ell_{0}^{2} K_{\nu}} \text {. }
$$

In Eq. (30), the $\left\{f_{0}\right\}$ is only the amplitude of $\left\{f_{\mathrm{P}}\right\}$. The direction of $f_{0}$ depends on $x_{\mathrm{P}}$ and it is determined with the signs conventions.

The problem is to determine the number of PZTs and such their distribution in order to the value of $f_{0}$ should attain its minimum. It leads to the maximum effectiveness of the reduction. Because of the value $I_{\nu ; \mathrm{E}} \neq 0$ and it is constant, only $K_{\nu}$ has the influence on the effectiveness of the p-reduction. Under circumstances given above, it comes up to the determination of $n_{\mathrm{P}}$ and $x_{\mathrm{P}}$.

The problem was solved in [3] based on the heuristic reasons. The PZTs are bonded on the beam sub-regions in which the curvatures take their maximum and the highest and lowest values. This is so-called QO distribution of the PZTs and it is described with $x_{Q} \equiv x_{\mathrm{P}}$ points and the $n_{Q} \equiv n_{\mathrm{P}}$ number of the PZT. It is proved in [3] that QO-distribution of the PZTs provides the maximum effectiveness of the p-reduction.

\section{Analytical method of the PZTs distribution}

The aim of the current section is to work out of the analytical method, which will describe such distribution of the PZTs in order to assure the maximum of the p-reduction effectiveness of the separate beam modes. It is expected that the analytical method will confirm the QO-distribution which has been found with heuristic method [3]. Therefore the assumptions are the same like in heuristic method, i.e. $n_{\mathrm{Q}}$ number of PZTs is assumed.

Let the distribution of PZTs be marked with the set of coordinates $\left\{x_{\mathrm{P}}^{\prime}\right\}$ for the moment; these are exactly these coordinates which are looked for. The starting equation is Eq. (30) via (29) one. Hence instead of Eq. (29) is

$$
\begin{aligned}
& K_{\nu}^{\prime}=\sum_{\mathrm{P}} \kappa_{\nu}\left(x_{\mathrm{P}}^{\prime}\right)=\kappa_{\nu}\left(x_{1}^{\prime}\right)+\kappa_{\nu}\left(x_{2}^{\prime}\right)+\ldots \\
& \quad+\kappa_{\nu}\left(x_{\mathrm{P}}^{\prime}\right)+\ldots+\kappa_{\nu}\left(x_{n_{\mathrm{P}}}^{\prime}\right) .
\end{aligned}
$$

Now, the value of $f_{0}$ is calculated from the formula, cf. Eq. (30),

$$
f_{0}=-\frac{I_{v ; E}}{\ell_{0}^{2} K_{\nu}^{\prime}}=-\frac{I_{v ; E}}{\ell_{0}^{2} \sum_{\mathrm{P}} \kappa_{\nu}\left(x_{\mathrm{P}}^{\prime}\right)} .
$$

The signs of $\kappa_{\nu}\left(x_{\mathrm{P}}^{\prime}\right)$ at separate points $x_{\mathrm{P}}^{\prime}$, have an influence on the sign of $f_{0}$; see signs convention in [3]. If the denominator in Eq. (32) has a maximum value, the $f_{0}$ takes a minimum one. Note, that $\kappa_{\nu}(x)$ is the function which changes the sign. So it is appropriate to search the points $x_{\mathrm{P}}^{\prime}$ which assure the extreme of the denominate, not maximum only. The function $\kappa_{\nu}(x)$ can have the extremum only at points $x_{\mathrm{P}}^{\prime}$, at which $D_{x} \kappa_{\nu}\left(x_{\mathrm{P}}^{\prime}\right)$ is equal

where 
to zero or $D_{x} \kappa_{\nu}\left(x_{\mathrm{P}}^{\prime}\right)$ does not exist ([21] Vol. 1, p. 241). Hence, a necessary condition for an extremum value is

$$
D_{x} \kappa_{\nu}=0 .
$$

From the condition (33), a set of stationary points $\left\{x_{\mathrm{P}}^{\prime}\right\}$ is obtained. For separate value $\nu, \nu=1,2,3$, the points $\left\{x_{\mathrm{P}}^{\prime}\right\}$ are depicted in Figs. 4b-6b (briefly - parts b) and they are marked with " $=$ ". The values of $\left\{x_{\mathrm{P}}^{\prime}\right\}$ are placed in Table.

Analytical results.

TABLE

\begin{tabular}{c|c|c|c|c|c}
\hline \hline$\nu$ & $\mathrm{P}$ & $\left\{x_{\mathrm{P}}^{\prime}\right\}$ & $\left\{x_{\mathrm{Q}}\right\}$ & $\kappa_{\nu}\left(x_{\mathrm{Q}}\right)$ & $f_{0}$ \\
\hline \multirow{2}{*}{1} & 1 & & 0 & 371.7 & 0.2583 \\
& 2 & $\ell$ & & 0 & \\
\hline \multirow{3}{*}{$\mathrm{s} 2$} & 1 & & 0 & $2.468 \times 10^{4}$ & 0.0144 \\
& 2 & 0.1429 & 0.1429 & $-2.336 \times 10^{4}$ & 0.0144 \\
& 3 & $\ell$ & & 0 & \\
\hline & 1 & & 0 & $1.329 \times 10^{6}$ & 0.0022 \\
3 & 2 & 0.0831 & 0.0831 & $-1439 \times 10^{6}$ & 0.0022 \\
& 3 & 0.1914 & 0.1914 & $1.655 \times 10^{6}$ & 0.0022 \\
& 4 & $\ell$ & & 0 &
\end{tabular}

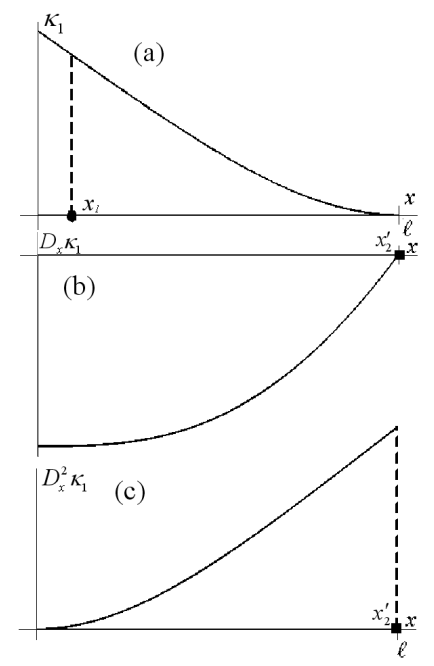

Fig. 4. (a) $\kappa_{1}(x)$, (b) $D_{x} \kappa_{1}(x)$, (c) $D_{x}^{2} \kappa_{1}(x)$.

The sufficient condition of existing extremum demands in order the function was determined on either side of the point $x_{\mathrm{P}}^{\prime}\left([21]\right.$ Vol. 1, p. 241) and $D_{x} \kappa_{\nu}(x)$ must change sign at this point (turning point); condition formulated in the first form is sufficient. This condition is expressed in the other form

$$
\left.D_{x}^{2} \kappa_{\nu}\right|_{x_{\mathrm{P}}^{\prime}} \neq 0 \text {. }
$$

The point $x_{\mathrm{P}}^{\prime}=\ell$ in parts $\mathrm{b}$ does not fulfill the above condition. This is because the function $\kappa_{\nu}(x)$ is not determined on either side of the point $x_{\mathrm{P}}^{\prime}=\ell$. So then this point should be omitted in future considerations. Since then it is easy to use the sufficient condition in the form (34). For this purpose the function $D_{x}^{2} \kappa_{\nu}(x)$

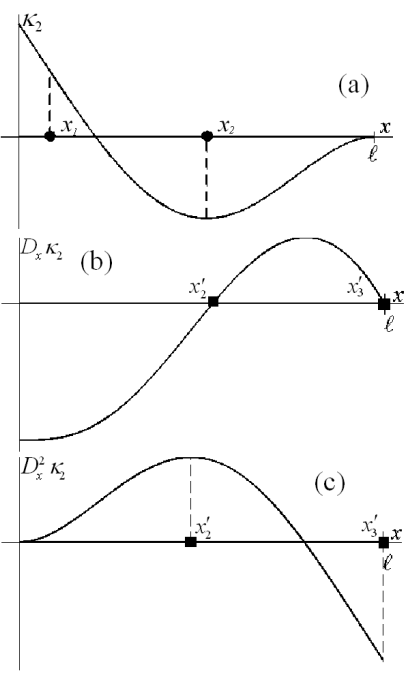

Fig. 5. (a) $\kappa_{2}(x)$, (b) $D_{x} \kappa_{2}(x)$, (c) $D_{x}^{2} \kappa_{2}(x)$.

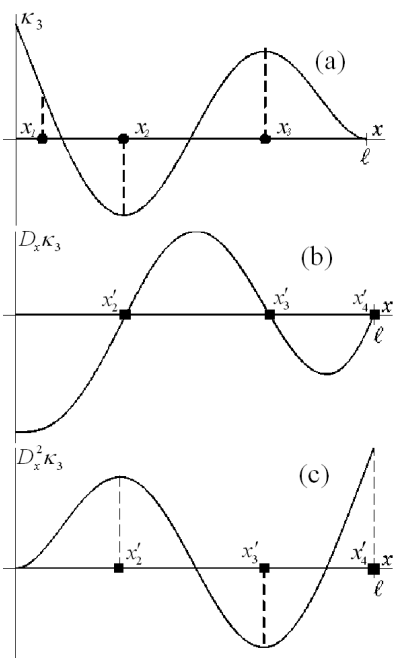

Fig. 6. (a) $\kappa_{3}(x)$, (b) $D_{x} \kappa_{3}(x)$, (c) $D_{x}^{2} \kappa_{3}(x)$.

is presented in Figs. 4c-6c (parts c). On these figures one can point out the points $\left\{x_{\mathrm{P}}^{\prime}\right\}$, at which the function $\kappa_{\nu}(x)$ has extremum and moreover, to decipher the sign of $D_{x}^{2} \kappa_{\nu}(x)$. But not only the points $\left\{x_{\mathrm{P}}^{\prime}\right\}$ play a major part here. Because of the form of Eq. (32), one still needs to consider the biggest and the lowest values of $\kappa_{\nu}(x)$; they are in hypothetical points $\left\{x_{\max }, x_{\min }\right\}$ ([21] Vol. 1, pp. $149 \div 252$ ). In order to find them, the values of $\kappa_{\nu}(x)$ at the stationary points $\left\{x_{\mathrm{P}}^{\prime}\right\}$ are calculated and they are compared to the values calculated at the end points of the interval $x \in[0, \ell]$; the results are tabulated in Table. As can be note, for all $\nu=1,2,3$, the curvature $\kappa_{\nu}(x)$ has the biggest value at the point $x=0=x_{\max }$. However, there is no the lowest value of $\kappa_{\nu}(x)$ at the point $x=\ell$. Hence, the point $x=0$ should be taken into consideration, but the point $x=\ell$ ought to pass over for some reasons: 1 - the necessary condition of the ex- 
tremum is not fulfilled, 2 - at this point and apart from $\kappa_{\nu}(\ell)=0$ the function $\kappa_{\nu}(x)$ does not taken its lowest value .

After considerations given above, the PZTs distributing points can be selected. The point $x_{\mathrm{P}}^{\prime}=\ell$ out of the stationary points should be rejected, whereas the point $x=0=x_{\max }$ should be connected. The selected points are described with $\left\{x_{\mathrm{P}}\right\}$ and they are marked by "•". in Figs. 4a-6a (parts a). Having to the disposal the values of $\left\{x_{\mathrm{P}}\right\}$, firstly, one can calculate the curvatures signs of $\kappa_{\nu}\left(x_{\mathrm{P}}\right)$. Next, based on Eq. (32), one can calculate the value and determine the sign of $f_{0}$.

\section{Numerical calculations}

The aim of the numerical tests is to confirm quantitatively the validation of the worked out analytical method. In numerical calculations the following data are assumed [3]: $\ell=0.27 \mathrm{~m}, a=0.025 \mathrm{~m}, h=0.001 \mathrm{~m}$, $J=\left(a h^{3}\right) / 12 \mathrm{~m}^{4}, E=2.05 \times 10^{11} \mathrm{~Pa}, \mu=1.94 \times 10^{-4} \mathrm{~s}$, $\rho_{b}=7.8 \times 10^{3} \mathrm{~kg} / \mathrm{m}^{3}, f_{\mathrm{E}}=0.02 \mathrm{~N} / \mathrm{m}$. The size of all PZTs is the same, i.e. $\ell_{1}=\ell_{2}=\ell_{3}=0.05 \ell$. Furthermore, all PZTs are excited by the same signal; it concerns the second and the third mode. The first PZTs has to be moved away from clamped side within necessary distance $\ell_{0}$. The quality results are depicted in Figs. 4-6, but the quantitative ones are collected in Table.

\section{Conclusions}

Based on the theoretical and numerical considerations, the following conclusions enumerated below may be formulated:

1. The PZTs distribution problem, assuring maximum of effectiveness of the p-reduction of the separate modes of the beam vibration, may be found analytically.

2. Analyzing the values of the points $\left\{x_{\mathrm{P}}\right\}$ it turns out that they are QO-point $\left\{x_{\mathrm{Q}}\right\}$ obtained earlier with heuristic method. So, the heuristic and analytical methods give the same results.

3. The algorithm of the analytical method can be formulated as follows

- to find the stationary points $\left\{x_{\mathrm{P}}^{\prime}\right\}$ of the curvature $\kappa_{\nu}(x)$,

- to select the points $\left\{x_{\mathrm{P}}^{\prime}\right\}$, at which the extremum of the curvature $\kappa_{\nu}(x)$ exists,

- to find the points $\left\{x_{\max }, x_{\min }\right\}$, at which the curvature $\kappa_{\nu}(x)$ has the highest and the lowest values,

- to select the $\left\{x_{\mathrm{P}}\right\}$-points of the PZTs distribution,

- to determine signs and to calculate the values both curvature $\kappa_{\nu}(x)$ and the force $f_{0}$ at the points $\left\{x_{\mathrm{P}}\right\}$. Such $f_{0}$ assures the maximum effectiveness of the p-reduction.
In the paper the analytical method of the PZTs distribution in vibration p-reduction problem was worked out for separate modes only. The similar research for general beam vibration and also for different, in geometric-technical regards, PZTs, may be more useful. The research on these problems is carried on.

\section{References}

[1] C.R. Fuller, S.J. Elliot, P.A. Nielsen, Active Control of Vibration, Academic Press, London 1997.

[2] C.H. Hansen, S.D. Snyder, Active Control of Noise and Vibration, E\&FN SPON, London 1997.

[3] A. Brański, M. Borkowski, S. Szela, in: Proc. XVI Conf. on Acoustic and Biomedical Engineering (IAB), Kraków-Zakopane (Poland) 2009, Eds. Z. Damijan, J. Wiciak, Katedra Mechaniki i Wibroakustyki AGH, Kraków 2009, p. 82.

[4] M.S. Kozień, J. Wiciak, in: Proc. Conf. Structural Acoustic - Waves - Biomedical Engineering, Eds. Z. Damijan, M. Iwaniec, J. Wiciak, Polish Acoustical Society, Kraków 2003, p. 47.

[5] M. Pietrzakowski, Monograph 204, Oficyna Wydawnicza Politechniki Warszawskiej, Warszawa 2004.

[6] P.M. Przybyłowicz, Monograph 197, Oficyna Wydawnicza Politechniki Warszawskiej, Warszawa 2002.

[7] J. Wiciak, Monograph 175, Akademia Górniczo-Hutnicza, Kraków 2008.

[8] J. Wiciak, Mol. Quant. Acoust. 25, 281 (2004).

[9] Z. Gosiewski, A. Koszewnik, in: Proc. Conf. Active Noise Vibration Control Method, Kraków, Ed. J. Kowal, 2007, Department of Process Control AGH, Kraków 2007, p. 69.

[10] M.S. Kozień, J. Wiciak, in: Proc. OSA'03, Eds. R. Bukowski, T. Pustelny, Gliwice 2003, p. 245 (in Polish).

[11] J. Wiciak, Acoust. Eng. 13, 145 (2004) (in Polish).

[12] M. Kozień, J. Wiciak, Mol. Quant. Acoust. 24, 97 (2003).

[13] M. Kozień, Monograph 331, Politechnika Krakowska, Kraków 2006 (in Polish).

[14] I. Bruant, L. Gallimard, S. Nikoukar, J. Sound Vib. 329, 1615 (2010).

[15] A. Brański, S. Szela, Arch. Control Sci. 17, 427 (2007).

[16] A. Brański, S. Szela, Arch. Acoust. 33, 413 (2008).

[17] A. Brański, S. Szela, in: Proc. 9th Conf. on Active Noise and Vibration Control Method (MARDiF), Kraków-Zakopane (Poland), Ed. J. Kowal, Department of Process Control AGH, Kraków 2009, p. 259.

[18] S. Kaliski, Vibration and Waves, PWN, Warszawa 1986 (in Polish).

[19] E. Kącki, Partial Differential Equations, WNT, Warszawa 1992 (in Polish).

[20] S. Ziemba, Vibration Analysis, Vol. II, PWN, Warszawa 1959 (in Polish).

[21] G.M. Fichtenholtz, Differential and Integral Calculus, PWN, Warszawa 1999 (in Polish). 